\title{
EXISTENCE OF WEAK SOLUTIONS FOR THREE-POINT BOUNDARY VALUE PROBLEMS OF KIRCHHOFF-TYPE
}

\author{
MOHAMMAD REZA HEIDARI TAVANI AND MEHDI KHODABAKHSHI
}

Received 25 February, 2019

\begin{abstract}
In this paper, we study the existence and multiplicity of solutions for a perturbed threepoint boundary value problem of Kirchhoff-type. The approach is based on variational methods and critical point theory. As applications of these methods, we get several multiplicity results for the problems under consideration.
\end{abstract}

2010 Mathematics Subject Classification: 35J20; 34B10; 34B15

Keywords: critical point theory, weak solution, variational method, Kirchhoff-type problem

\section{INTRODUCTION}

In this paper, we consider the following perturbed second-order Kirchhoff-type problem

$$
\left\{\begin{array}{l}
-K\left(\int_{a}^{b}\left|u^{\prime}(x)\right|^{2} d x\right) u^{\prime \prime}(x)=\lambda \alpha(x) f(x, u(x)), \quad x \in(a, b), \\
u(a)=0, u(b)=\beta u(\gamma)
\end{array}\right.
$$

where $\lambda$ is a positive parameter, $K:[0,+\infty[\rightarrow \mathbb{R}$ is a continuous function such that there exist positive numbers $m_{0}$ and $m_{1}$ with $m_{0} \leq K(x) \leq m_{1}$ for all $x \geq 0, a, b \in$ $\mathbb{R}$ with $a<b, f:[a, b] \times \mathbb{R} \rightarrow \mathbb{R}$ is a non-negative $L^{1}$-Carathéodory function, $\alpha \in$ $L^{\infty}([a, b]), \alpha(x) \geq 0$, for a.e. $x \in[a, b], \alpha \neq \equiv 0, \beta \in \mathbb{R}$ and $\gamma \in(a, b)$.

In recent years, existence and multiplicity results for this kinds of problems has been extensively studied (see $[1,2,4,9,15]$ and the references therein ).

For example, Gupta [15] under natural conditions on $f$ using degree-theoretic arguments, obtained existence and uniqueness theorems to the three-point nonlinear second-order boundary value problem

$$
\left\{\begin{array}{l}
u^{\prime \prime}=f\left(x, u(x), u^{\prime}(x)\right)-e(x), \quad 0<x<1, \\
u(0)=0, u(\eta)=u(1)
\end{array}\right.
$$

where $f:[0,1] \times \mathbb{R}^{2} \rightarrow \mathbb{R}$ is a function satisfying Carathéodorys conditions, $e$ : $[0,1] \rightarrow \mathbb{R}$ is a function in $L^{1}(0,1)$ and $\eta \in(0,1)$. 
Authors in [4] based upon Leggett-Williams fixed-point theorem, provided conditions for the existence of three positive solutions to the nonlinear boundary value problem

$$
\left\{\begin{array}{l}
u^{\prime \prime}+f(t, u)=0, \\
u(0)=0, \operatorname{au}(\eta)=u(1)
\end{array} \quad t \in(0,1),\right.
$$

where $0<\eta<1,0<a$ and $a \eta<1$. Also $f:[0,1] \times[0, \infty) \rightarrow[0, \infty)$ is a continuous function and $f(t,$.$) does not vanish identically on any subset of [0,1]$ with positive measure. Recently, Lin in [9] by using variational methods and a three-critical-point theorem, considered the existence of at least three solutions for problem (1.2). In [2] authors, based on variational methods and a critical point theorem established the existence of at least one weak solution for problem

$$
\left\{\begin{array}{l}
-K\left(\int_{a}^{b}\left|u^{\prime}(t)\right|^{2} d t\right) u^{\prime \prime}(t)=f(t, u(t))+h(u(t)), \quad t \in(a, b), \\
u(a)=0, u(b)=\alpha u(\eta)
\end{array}\right.
$$

where $K:[0,+\infty[\rightarrow \mathbb{R}$ is a continuous function such that there exist positive numbers $m$ and $M$ with $m \leq K(x) \leq M$ for all $x \geq 0, a, b \in \mathbb{R}$ with $a<b, f:[a, b] \times \mathbb{R} \rightarrow$ $\mathbb{R}$ is an $L^{1}$-Carathéodory function, $h: \mathbb{R} \rightarrow \mathbb{R}$ is a Lipschitz continuous function with the Lipschitz constant $L>0, \alpha \in \mathbb{R}$ and $\eta \in(a, b)$. Different types of Kirchhoff equation are expressed in [16]. It was proposed as an extension of the classical D'Alembert's wave equation for free vibrations of elastic strings. The Kirchhoff's model takes into account the length changes of the string produced by transverse vibrations. Some interesting results can be found, for example in $[3,8,11,16]$. Many researchers have studied the problems of Kirchhoff-type. We refer the reader to the papers $[5-7,10,17]$ and the references therein.

In this paper, using two kinds of critical points theorems obtained in [12], we establish the existence of weak solutions for the problem (1.1). The paper is organized as follows.

In section 2 we establish all the preliminary results that we need, and in section 3 we present our main results.

\section{PRELIMINARIES}

Let us recall some basic concepts.

Definition 1. A function $f:[a, b] \times \mathbb{R} \rightarrow \mathbb{R}$ is said to be a Carathéodory function if

$\left(C_{1}\right)$ the function $x \rightarrow f(x, t)$ is measurable for every $t \in \mathbb{R}$;

$\left(C_{2}\right)$ the function $t \rightarrow f(x, t)$ is continuous for a.e. $x \in[a, b]$.

And $f:[a, b] \times \mathbb{R} \rightarrow \mathbb{R}$ is said to be a $L^{1}$-Carathéodory function if, in addition to conditions $\left(C_{1}\right)$ and $\left(C_{2}\right)$, the following condition is also satisfied :

$\left(C_{3}\right)$ for every $\rho>0$ there is function $l_{\rho} \in L^{1}([a, b])$ such that $\sup _{|t| \leq \rho}|f(x, t)| \leq$ $l_{\rho}(x)$ for almost every $x \in[a, b]$. 
Denote

$$
X:=\left\{u \in W^{1,2}(a, b) \mid u(a)=0, u(b)=\beta u(\gamma)\right\},
$$

endowed with the norm

$$
\|u\|:=\left(\int_{a}^{b}\left|u^{\prime}(x)\right|^{2} d x\right)^{1 / 2}
$$

Theorem 1 ([9], Theorem 3.2). X is a separable and reflexive real Banach space.

The following lemma is useful for proving our main result.

Lemma 1 ([2], Lemma 2.4). For all $u \in X$, we have

$$
\max _{x \in[a, b]}|u(x)| \leq \frac{(1+|\beta|) \sqrt{b-a}}{2}\|u\| .
$$

Remark 1. If $\left\{u_{n}\right\}_{n \in \mathbb{N}}$ is a bounded sequence in $X$, then from the compact embedding $X \hookrightarrow C([a, b])$ it has a subsequence that pointwise converges to some $u \in X$ (it comes from the definition of compact embedding). Also since $X$ is reflexive space then there exist a subsequence that weakly converges in $X$ (see [14]-Theorem 3.18) and so according to continuous embedding $X \rightarrow L^{\infty}([a, b])$ weakly converges in $L^{\infty}([a, b])$.

Corresponding to the functions $f$ and $K$ we introduce the functions $F:[a, b] \times$ $\mathbb{R} \rightarrow \mathbb{R}$ and $\widetilde{K}:[0, \infty) \rightarrow \mathbb{R}$ respectively, as follows

and

$$
F(x, t)=\int_{0}^{t} f(x, \xi) d \xi \text { for all }(x, t) \in[a, b] \times \mathbb{R}
$$

Moreover, set

$$
\widetilde{K}(t)=\int_{0}^{t} K(\xi) d \xi \text { for all } t \geq 0
$$

$$
F^{\theta}=\int_{a}^{b} \sup _{|\xi| \leq \theta} F(x, \xi) d x \text { for all } \theta>0 .
$$

Let $\Phi, \Psi: X \rightarrow \mathbb{R}$ be defined by

$$
\Phi(u)=\frac{1}{2} \widetilde{K}\left(\|u\|^{2}\right)
$$

and

for every $u \in X$.

$$
\Psi(u)=\int_{a}^{b} \alpha(x) F(x, u(x)) d x
$$


We observe that

$$
\frac{m_{0}}{2}\|u\|^{2} \leq \Phi(u) \leq \frac{m_{1}}{2}\|u\|^{2}
$$

for every $u \in X . \Psi$ is a differentiable functional whose differential at the point $u \in X$ is

$$
\Psi^{\prime}(u)(v)=\int_{a}^{b} \alpha(x) f(x, u(x)) v(x) d x,
$$

and, $\Phi$ is continuously Gâteaux differentiable functional whose differential at the point $u \in X$ is

$$
\Phi^{\prime}(u)(v)=K\left(\int_{a}^{b}\left|u^{\prime}(x)\right|^{2}\right)\left(\int_{a}^{b} u^{\prime}(x) v^{\prime}(x) d x\right)
$$

for every $v \in X$.

Definition 2. Let $\Phi$ and $\Psi$ be defined as above and put $I_{\lambda}=\Phi-\lambda \Psi$. We say that $u \in X$ is a critical point of $I_{\lambda}$ when $I_{\lambda}^{\prime}(u)=0_{\left\{X^{*}\right\}}$, that is, $I_{\lambda}^{\prime}(u)(v)=$ $\Phi^{\prime}(u)(v)-\lambda \Psi^{\prime}(u)(v)=0$ for all $v \in X$.

Definition 3. A function $u \in X$ is a weak solution to the problem (1.1) if

$$
K\left(\int_{a}^{b}\left|u^{\prime}(x)\right|^{2}\right)\left(\int_{a}^{b} u^{\prime}(x) v^{\prime}(x) d x\right)-\lambda \int_{a}^{b} \alpha(x) f(x, u(x)) v(x) d x=0
$$

for every $v \in X$.

Remark 2. We observe that the weak solutions of the problem (1.1) are exactly the solutions of the equation $I_{\lambda}^{\prime}(u)(v)=0$. Also if $\alpha$ and $f$ are, in addition, continuous functions, then each weak solution of (1.1) is a classical solution.

Lemma 2. If $u_{0} \not \equiv 0$ is a weak solution for problem (1.1) then $u_{0}$ is non-negative.

Proof. From remark 2 one has, $I_{\lambda}^{\prime}\left(u_{0}\right)(v)=0$ for all $v \in X$. Chossing $v(x)=$ $\max \left\{-u_{0}(x), 0\right\}$ and let $E=\left\{x \in[a, b]: u_{0}(x)<0\right\}$. Then we have

$$
\begin{gathered}
K\left(\int_{E \cup E^{c}}\left|u_{0}^{\prime}(x)\right|^{2} d x\right)\left(\int_{E \cup E^{c}} u_{0}^{\prime}(x) v^{\prime}(x) d x\right) \\
=\lambda \int_{a}^{b} \alpha(x) f\left(x, u_{0}(x)\right) v(x) d x \geq 0
\end{gathered}
$$

that is

$$
-m_{1} \int_{E} v^{\prime}(x) v^{\prime}(x) d x \geq 0
$$

which means that $-m_{1}\|v\|^{2} \geq 0$ and one has $\|v\|=0$ and therefore $v=0$. Hence $-u_{0} \leq 0$, that is, $u_{0} \geq 0$ and the proof is complete. 
Definition 4 (see [13]). Fix $r \in]-\infty,+\infty$ ] . A Gâtuax differentiable function $I$ satisfies the Palais-Smale condition cut off upper at $r$ (in short $(P S)^{[r]}$-condition) if any sequence $\left\{u_{n}\right\}$ such that

(a) $\left\{I\left(u_{n}\right)\right\}$ is bounded,

(b) $\lim _{n \rightarrow+\infty}\left\|I^{\prime}\left(u_{n}\right)\right\|_{X^{*}}=0$,

(c) $\Phi\left(u_{n}\right)<r \quad \forall n \in \mathbb{N}$,

has a convergent subsequence.

Definition 5. A Gâtuax differentiable function $I$ satisfies the Palais-Smale condition (in short $(P S)$-condition) if any sequence $\left\{u_{n}\right\}$ such that

(a) $\left\{I\left(u_{n}\right)\right\}$ is bounded,

(b) $\lim _{n \rightarrow+\infty}\left\|I^{\prime}\left(u_{n}\right)\right\|_{X^{*}}=0, \quad \forall n \in \mathbb{N}$,

has a convergent subsequence.

Our main tools are the following critical point theorems.

Theorem 2 ([12], Theorem 2.3). Let $X$ be a real Banach space, and let $\Phi, \Psi$ : $X \longrightarrow \mathbb{R}$ be two continuously Gâteaux differentiable functionals such that $\inf _{X} \Phi=$ $\Phi(0)=\Psi(0)=0$.

Assume that there are $r \in \mathbb{R}$ and $\tilde{u} \in X$, with $0<\Phi(\tilde{u})<r$, such that

$$
\frac{\sup _{u \in \Phi^{-1}(]-\infty, r[)} \Psi(u)}{r}<\frac{\Psi(\tilde{u})}{\Phi(\tilde{u})}
$$

and , for each $\lambda \in] \frac{\Phi(\tilde{u})}{\Psi(\tilde{u})}, \frac{r}{\sup _{u \in \Phi^{-1}(]-\infty, r[)} \Psi(u)}[$ the functional $\Phi-\lambda \Psi$ satisfies the $(P S)^{[r]}$-condition. Then, for each $\left.\lambda \in\right] \frac{\Phi(\tilde{u})}{\Psi(\tilde{u})}, \frac{r}{\sup _{u \in \Phi^{-1}(]-\infty, r[)} \Psi(u)}[$ there is $u_{\lambda} \in \Phi^{-1}(] 0, r[)$ (hence $u_{\lambda} \neq 0$ ) such that $I_{\lambda}\left(u_{\lambda}\right)<I_{\lambda}(u)$ for all $u \in \Phi^{-1}(] 0, r[)$ and $I_{\lambda}^{\prime}\left(u_{\lambda}\right)=0$.

Theorem 3 ([12], Theorem 3.2). Let $X$ be a real Banach space, and let $\Phi, \Psi$ : $X \longrightarrow \mathbb{R}$ be two continuously Gâteaux differentiable functionals such that $\Phi$ is bounded from below and

$\Phi(0)=\Psi(0)=0$. Fix $r>0$ such that $\sup _{u \in \Phi^{-1}(]-\infty, r[)} \Psi(u)<+\infty$ and assume that for each $\lambda \in] 0, \frac{r}{\sup _{u \in \Phi^{-1}(]-\infty, r[)} \Psi(u)}[$ the functional $\Phi-\lambda \Psi$ satisfies the $(P S)$-condition and it is unbounded from below. Then, for each

$$
\lambda \in] 0, \frac{r}{\sup _{u \in \Phi^{-1}(]-\infty, r[)} \Psi(u)}[
$$

the functional $I_{\lambda}$ admits two distinct critical points. 
Now we present two propositions that will be needed to prove the main theorems of this paper.

Proposition 1. Take $I_{\lambda}=\Phi-\lambda \Psi$ as in the definition 2. Then $I_{\lambda}$ satisfies the $(P S)^{[r]}$-condition for any $r>0$.

Proof. Consider sequence $\left\{u_{n}\right\} \subseteq X$ such that $\left\{I_{\lambda}\left(u_{n}\right)\right\}$ is bounded, $\lim _{n \rightarrow+\infty}\left\|I_{\lambda}^{\prime}\left(u_{n}\right)\right\|_{X^{*}}=0$ and $\Phi\left(u_{n}\right)<r, \forall n \in \mathbb{N}$. Since $\Phi\left(u_{n}\right)<r$, then from (2.4) we see that, $\left\{u_{n}\right\}$ is bounded in $X$. Therefore passing to a subsequence if necessary we can assume that $u_{n}(x) \rightarrow u(x)$, and there is $s>0$ such that $\left|u_{n}(x)\right| \leq s$ for all $x \in[a, b]$ and for all $n \in \mathbb{N}$ and also $\left\{u_{n}\right\}$ weakly converges to $u$ in $L^{\infty}([a, b])$ ( see Remark 1). Now according to Hölder's inequality and Lebesque's Dominated Convergence Theorem, Since $\alpha(x) f\left(x, u_{n}(x)\right) \leq \alpha(x)$. $\max _{|\xi| \leq s} f(x, \xi) \in L^{1}([a, b])$ for all $n \in \mathbb{N}$ and $f\left(x, u_{n}(x)\right) \rightarrow f(x, u(x))$ for a.e. $x \in[a, b]\left(f\right.$ is $L^{1}$-Carathéodory function), one has $\alpha f\left(x, u_{n}\right)$ is strongly converging to $\alpha f(x, u)$ in $L^{1}([a, b])$. Now since $u_{n} \rightarrow u$ in $L^{\infty}([a, b])$ and $\alpha f\left(x, u_{n}\right) \rightarrow \alpha f(x, u)$ in $L^{1}([a, b]) \subseteq\left(L^{\infty}([a, b])\right)^{*}$ then from [[14],proposition 3.5(iv)], one has

$$
\lim _{n \rightarrow+\infty} \int_{a}^{b} \alpha(x) f\left(x, u_{n}(x)\right)\left(u_{n}(x)-u(x)\right) d x=0 .
$$

From $\lim _{n \rightarrow+\infty}\left\|I_{\lambda}^{\prime}\left(u_{n}\right)\right\|_{X}=0$, there exists a sequence $\left\{\varepsilon_{n}\right\}$, with $\varepsilon_{n} \rightarrow 0^{+}$, such that

$$
\begin{aligned}
& \mid K\left(\int_{a}^{b}\left|u_{n}^{\prime}(x)\right|^{2}\right)\left(\int_{a}^{b} u_{n}^{\prime}(x) v^{\prime}(x) d x\right) \\
& -\lambda \int_{a}^{b} \alpha(x) f\left(x, u_{n}(x)\right) v(x) d x \mid \leq \varepsilon_{n},
\end{aligned}
$$

, for all $n \in \mathbb{N}$ and for all $v \in X$ with $\|v\| \leq 1$. Taking into account $v(x)=\frac{u_{n}(x)-u(x)}{\left\|u_{n}-u\right\|}$, from (2.8) one has

$$
\begin{gathered}
\mid K\left(\int_{a}^{b}\left|u_{n}^{\prime}(x)\right|^{2}\right) \int_{a}^{b} u_{n}^{\prime}(x)\left(u_{n}^{\prime}(x)-u^{\prime}(x)\right) d x \\
-\lambda \int_{a}^{b} \alpha(x) f\left(x, u_{n}(x)\right)\left(u_{n}(x)-u(x)\right) d x \mid \leq \varepsilon_{n}\left\|u_{n}-u\right\|
\end{gathered}
$$

for all $n \in \mathbb{N}$. Now according to inequality $|a||b| \leq \frac{1}{2}|a|^{2}+\frac{1}{2}|b|^{2}$ one has

$$
\begin{gathered}
K\left(\int_{a}^{b}\left|u_{n}^{\prime}(x)\right|^{2}\right) \int_{a}^{b} u_{n}^{\prime}(x)\left(u_{n}^{\prime}(x)-u^{\prime}(x)\right) d x= \\
K\left(\int_{a}^{b}\left|u_{n}^{\prime}(x)\right|^{2}\right)\left(\int_{a}^{b}\left|u_{n}^{\prime}(x)\right|^{2} d x-\int_{a}^{b} u_{n}^{\prime}(x) u^{\prime}(x) d x\right) \geq
\end{gathered}
$$




$$
\begin{gathered}
m_{0}\left(\left\|u_{n}\right\|^{2}-\int_{a}^{b}\left(\frac{1}{2}\left|u_{n}^{\prime}(x)\right|^{2}+\frac{1}{2}\left|u^{\prime}(x)\right|^{2}\right) d x\right)= \\
m_{0}\left(\left\|u_{n}\right\|^{2}-\frac{1}{2}\left\|u_{n}\right\|^{2}-\frac{1}{2}\|u\|^{2}\right)=m_{0}\left(\frac{1}{2}\left\|u_{n}\right\|^{2}-\frac{1}{2}\|u\|^{2}\right) .
\end{gathered}
$$

Hence from (2.9), we have

$$
\begin{aligned}
m_{0}\left(\frac{1}{2}\left\|u_{n}\right\|^{2}-\frac{1}{2}\|u\|^{2}\right) & \leq \lambda \int_{a}^{b} \alpha(x) f\left(x, u_{n}(x)\right)\left(u_{n}(x)-u(x)\right) d x \\
& +\varepsilon_{n}\left\|u_{n}-u\right\|
\end{aligned}
$$

that is,

$$
\begin{gathered}
\frac{m_{0}}{2}\left\|u_{n}\right\|^{2} \leq \frac{m_{0}}{2}\|u\|^{2} \\
+\lambda \int_{a}^{b} \alpha(x) f\left(x, u_{n}(x)\right)\left(u_{n}(x)-u(x)\right) d x+\varepsilon_{n}\left\|u_{n}-u\right\| .
\end{gathered}
$$

Now, according to (2.7) and (2.10) when $\varepsilon_{n} \rightarrow 0^{+}$, we have,

$$
\limsup _{n \rightarrow+\infty}\left\|u_{n}\right\| \leq\|u\| \text {. }
$$

Thus [14], Proposition 3.32 ensures that $u_{n} \rightarrow u$, strongly in $X$ and the proof is complete.

Proposition 2. Assume that there are $M>0$ and $\theta$ with $\theta m_{0}>2 m_{1}$ such that

$$
0<\theta F(x, \xi) \leq \xi f(x, \xi)
$$

for all $\xi \geq M$ and $x \in[a, b]$. Then $I_{\lambda}=\Phi-\lambda \Psi$ satisfies the $(P S)$-condition and it is unbounded from below.

Proof. First we prove that $I_{\lambda}$ satisfies $(P S)$-condition for every $\lambda>0$. For this purpose we will prove that for arbitrary sequence $\left\{u_{n}\right\} \subset X$ satisfying

$$
\begin{gathered}
\left|I_{\lambda}\left(u_{n}\right)\right| \leq D \text { for some } D>0 \text { and for all } n \in \mathbb{N}, \\
I_{\lambda}^{\prime}\left(u_{n}\right) \rightarrow 0 \text { in } X^{*} \text { as } n \rightarrow \infty,
\end{gathered}
$$

contains a convergent subsequence. Let $\bar{u}_{n}(x)=\max \left\{0,-u_{n}(x)\right\}$. From (2.13) we have

$$
\left|I_{\lambda}^{\prime}\left(u_{n}\right)(v)\right| \leq \varepsilon_{n}\|v\|
$$

for all $v \in X$ with $\varepsilon_{n} \rightarrow 0^{+}$. Hence $\left|I_{\lambda}^{\prime}\left(u_{n}\right)\left(\bar{u}_{n}\right)\right| \leq \varepsilon_{n}\left\|\bar{u}_{n}\right\|$, that is,

$$
\begin{gathered}
\mid K\left(\int_{a}^{b}\left|u_{n}^{\prime}(x)\right|^{2}\right)\left(\int_{a}^{b} u_{n}^{\prime}(x) \bar{u}_{n}^{\prime}(x) d x\right) \\
-\lambda \int_{a}^{b} \alpha(x) f\left(x, u_{n}(x)\right) \bar{u}_{n}(x) d x \mid \leq \varepsilon_{n}\left\|\bar{u}_{n}\right\| .
\end{gathered}
$$


Therefore

$$
m_{0}\left\|\bar{u}_{n}\right\|^{2}+\lambda \int_{a}^{b} \alpha(x) f\left(x, u_{n}(x)\right) \bar{u}_{n}(x) d x \leq \varepsilon_{n}\left\|\bar{u}_{n}\right\| .
$$

Hence $m_{0}\left\|\bar{u}_{n}\right\|^{2} \leq \varepsilon_{n}\left\|\bar{u}_{n}\right\|$ and so $\left\|\bar{u}_{n}\right\| \leq \frac{\varepsilon_{n}}{m_{0}}$. Therefore $\left\{\bar{u}_{n}\right\}$ is strongly converges to 0 in $X$ and so it is bounded in $X$. Thus according to compact embedding $X \rightarrow C([a, b])$ it is bounded in $C([a, b])$ and hence $0 \leq \bar{u}_{n}(x) \leq L$ for some $L \geq 0$ and for all $x \in[a, b]$ and one has,

$$
u_{n}(x) \geq-L
$$

for all $x \in[a, b]$ and for all $n \in \mathbb{N}$. Now we prove that $\left\{u_{n}\right\}$ is bounded in $X$. For this end from (2.14), we have

$$
-I_{\lambda}^{\prime}\left(u_{n}\right)\left(u_{n}\right) \leq \varepsilon_{n}\left\|u_{n}\right\|
$$

for all $n \in \mathbb{N}$ with $\varepsilon_{n} \rightarrow 0^{+}$. Now we have

$$
\begin{gathered}
I_{\lambda}\left(u_{n}\right)-\frac{1}{\theta} I_{\lambda}^{\prime}\left(u_{n}\right)\left(u_{n}\right)=\left(\frac{m_{0}}{2}-\frac{m_{1}}{\theta}\right)\left\|u_{n}\right\|^{2} \\
+\frac{\lambda}{\theta} \int_{a}^{b} \alpha(x)\left[f\left(x, u_{n}(x)\right) u_{n}(x)-\theta F\left(x, u_{n}(x)\right)\right] d x .
\end{gathered}
$$

From (2.11) one has

$$
\int_{u_{n}(x) \geq M} \alpha(x)\left[f\left(x, u_{n}(x)\right) u_{n}(x)-\theta F\left(x, u_{n}(x)\right)\right] d x \geq 0 .
$$

On the other hand, in view of (2.15) we have

$$
\begin{aligned}
& \left|\int_{-L \leq u_{n}(x)<M} \alpha(x)\left[f\left(x, u_{n}(x)\right) u_{n}(x)-\theta F\left(x, u_{n}(x)\right)\right] d x\right| \\
& \quad \leq \int_{-L \leq u_{n}(x)<M} \alpha(x) \sup _{\xi \in[-L, M]}[f(x, \xi) \xi-\theta F(x, \xi)] d x .
\end{aligned}
$$

Hence from (2.17) we have

$$
\begin{gathered}
I_{\lambda}\left(u_{n}\right)-\frac{1}{\theta} I_{\lambda}^{\prime}\left(u_{n}\right)\left(u_{n}\right) \geq\left(\frac{m_{0}}{2}-\frac{m_{1}}{\theta}\right)\left\|u_{n}\right\|^{2} \\
-\frac{\lambda}{\theta} \int_{-L \leq u_{n}(x)<M} \alpha(x) \sup _{\xi \in[-L, M]}[f(x, \xi) \xi-\theta F(x, \xi)] d x .
\end{gathered}
$$

Taking into account (2.12),(2.16), from (2.18) one has

$$
\left(\frac{m_{0}}{2}-\frac{m_{1}}{\theta}\right)\left\|u_{n}\right\|^{2}-\frac{\lambda}{\theta} \int_{-L \leq u_{n}(x)<M} \alpha(x) \sup _{\xi \in[-L, M]}[f(x, \xi) \xi-\theta F(x, \xi)] d x
$$


that is,

$$
\leq D+\frac{\varepsilon_{n}}{\theta}\left\|u_{n}\right\|
$$

$$
\begin{gathered}
\left(\frac{m_{0}}{2}-\frac{m_{1}}{\theta}\right)\left\|u_{n}\right\|^{2} \leq \\
D+\frac{\varepsilon_{n}}{\theta}\left\|u_{n}\right\|+\frac{\lambda}{\theta} \int_{-L \leq u_{n}(x)<M} \alpha(x) \sup _{\xi \in[-L, M]}[f(x, \xi) \xi-\theta F(x, \xi)] d x
\end{gathered}
$$

and thus, (2.19) ensures that $\left\{u_{n}\right\}$ is bounded in $X$. Finally, as the argument used to prove Proposition $1,\left\{u_{n}\right\}$ admits a convergent subsequence and so $I_{\lambda}$ satisfies $(P S)$ condition.

Now, from (2.11) we will show that there are positive constants $c_{1}$ and $c_{2}$ such that

$$
F(x, \xi) \geq c_{1} \xi^{\theta}-c_{2}
$$

for all $\xi \geq 0$ and $x \in(a, b)$. Put $\phi_{\xi}(s):=F(x, s \xi), \forall s>0$. From (2.11), for all $\xi>$ $M$ and $x \in(a, b)$ we have

$$
0<\theta \phi_{\xi}(s)=\theta F(x, s \xi) \leq s \xi . f(x, s \xi)=s \phi_{\xi}^{\prime}(s), \forall s>\frac{M}{\xi} .
$$

Hence,

Therefore, we have

$$
\int_{\frac{M}{\xi}}^{1} \frac{\phi_{\xi}^{\prime}(s)}{\phi_{\xi}(s)} d s \geq \int_{\frac{M}{\xi}}^{1} \frac{\theta}{S} d s .
$$

Now, in view of (2.21) one has

$$
\phi_{\xi}(1) \geq \phi_{\xi}\left(\frac{M}{\xi}\right)\left(\frac{\xi}{M}\right)^{\theta} \text {. }
$$

$$
F(x, \xi) \geq \frac{A}{M^{\theta}} \xi^{\theta}
$$

where $A=\inf _{x \in(a, b)} F(x, M)$. On the other hand for every $0 \leq \xi \leq M$ we have $F(x, \xi) \leq b(x)$, where $b(x)=\sup _{0 \leq \xi \leq M} F(x, \xi)$. Therefore for every $0 \leq \xi \leq M$ we have

$$
F(x, \xi) \geq-b(x) .
$$

Thus, (2.20) is proved according to (2.22) and (2.23).

Fixed $u_{0} \in X-\{0\}$, and suppose that $u_{0} \geq 0$. For each $t>1$, we have

$$
I_{\lambda}\left(t u_{0}\right)=\frac{1}{2} \widetilde{K}\left(\left\|t u_{0}\right\|^{2}\right)-\lambda \int_{a}^{b} \alpha(x) . F\left(x, t u_{0}\right) d x .
$$

Taking into account (2.20), one has

$$
I_{\lambda}\left(t u_{0}\right) \leq \frac{m_{1} t^{2}}{2}\left\|u_{0}\right\|^{2}-\lambda c_{1} t^{\theta} \int_{a}^{b} \alpha(x) \cdot u_{0}{ }^{\theta} d x+\lambda c_{2} \int_{a}^{b} \alpha(x) d x
$$


and since $\theta>2$ (it comes from condition $\theta m_{0}>2 m_{1}$ ), this condition guarantees that $I_{\lambda}$ is unbounded from below.

\section{MAIN RESULTS}

In this section we formulate our main results. Put

$$
\begin{gathered}
Z:=\frac{1}{\gamma-a}+\frac{2(\beta-1)^{2}}{b-\gamma}, \\
\tau:=\sqrt{\frac{m_{0}}{m_{1}}}
\end{gathered}
$$

and

$$
\Theta:=\frac{2 m_{0}}{(1+|\beta|)^{2}(b-a)} .
$$

Now we express the main results.

Theorem 4. Assume that there exist two positive constants $\delta$ and $\theta$ with

$$
\delta<\frac{2 \tau \theta}{(1+|\beta|) \sqrt{Z(b-a)}}
$$

such that

(i) $\frac{F^{\theta}}{\theta^{2}}<\frac{2 \Theta \int_{\gamma}^{\frac{b+\gamma}{2}} \alpha(x) F(x, \delta) d x}{\|\alpha\|_{\infty} m_{1} Z \delta^{2}}$,

where $\|\alpha\|_{\infty}=\|\alpha\|_{L^{\infty}}$. Then, for each

$$
\lambda \in \Lambda:=] \frac{m_{1} Z \delta^{2}}{2 \int_{\gamma}^{\frac{b+\gamma}{2}} \alpha(x) F(x, \delta) d x}, \frac{\Theta \theta^{2}}{\|\alpha\|_{\infty} F^{\theta}}[,
$$

the problem (1.1) admits at least one non-trivial and non-negative weak solution $u_{\lambda} \in X$ such that $\left|u_{\lambda}(x)\right|<\theta$ for all $x \in[a, b]$.

Proof. Our aim is to apply Theorem 2, to problem (1.1). Fix $\lambda$, as in the conclusion. Take $X, \Phi$ and $\Psi$ as in the previous section. We observe that the regularity assumptions of Theorem 2 on $\Phi$ and $\Psi$ are satisfied. Also according to Proposition 1, the functional $I_{\lambda}$ satisfies the $(P S)^{[r]}$-condition for all $r>0$. 
Put

$$
r:=\Theta \theta^{2}
$$

and

$$
w(x):= \begin{cases}\frac{\delta}{\gamma-a}(x-a) & \text { if } x \in[a, \gamma) \\ \delta & \text { if } x \in\left[\gamma, \frac{b+\gamma}{2}\right] \\ \delta\left(\frac{2(\beta-1)}{b-\gamma} x-\frac{\beta(b+\gamma)-2 b}{b-\gamma}\right) & \text { if } x \in\left(\frac{b+\gamma}{2}, b\right] .\end{cases}
$$

We observe that $w \in X$ and

$$
\|w\|^{2}=\int_{a}^{b}\left|w^{\prime}(x)\right|^{2} d x=\left(\frac{1}{\gamma-a}+\frac{2(\beta-1)^{2}}{b-\gamma}\right) \delta^{2}=Z \delta^{2} .
$$

In particular, from (2.4) one has

$$
\frac{m_{0}}{2} Z \delta^{2} \leq \Phi(w) \leq \frac{m_{1}}{2} Z \delta^{2} .
$$

Therefore, from (3.4) one has $0<\Phi(w)<r$. Now for each $u \in X$ and bearing (2.1) in mind, we see that

$$
\begin{aligned}
\Phi^{-1}(]-\infty, r[) & =\{u \in X ; \Phi(u)<r\} \\
& \subseteq\left\{u \in X ; \frac{m_{0}}{2}\|u\|^{2}<r\right\} \\
& \subseteq\{u \in X ;|u(x)|<\theta \text { for each } x \in[a, b]\},
\end{aligned}
$$

and it follows that

$$
\begin{aligned}
\sup _{u \in \Phi^{-1}(]-\infty, r[)} \Psi(u) & =\sup _{u \in \Phi^{-1}(]-\infty, r[)}\left(\int_{a}^{b} \alpha(x) F(x, u(x)) d x\right) \\
& \leq \int_{a}^{b} \alpha(x) \sup _{|\xi| \leq \theta} F(x, \xi) d x \leq\|\alpha\|_{\infty} F^{\theta} .
\end{aligned}
$$

Hence, we have

$$
\frac{\sup _{u \in \Phi^{-1}(]-\infty, r[} \Psi(u)}{r} \leq \frac{\|\alpha\|_{\infty} F^{\theta}}{\Theta \theta^{2}}<\frac{1}{\lambda} .
$$

On the other hand, from (3.8) and since $\lambda \in \Lambda$, one has,

$$
\frac{\Psi(w)}{\Phi(w)} \geq \frac{\int_{a}^{b} \alpha(x) F(x, w(x)) d x}{\frac{m_{1}}{2} Z \delta^{2}} \geq \frac{\int_{\gamma}^{\frac{b+\gamma}{2}} \alpha(x) F(x, \delta) d x}{\frac{m_{1}}{2} Z \delta^{2}}>\frac{1}{\lambda} .
$$

Now from (3.9) and (3.10) we have,

$$
\frac{\sup _{u \in \Phi^{-1}(]-\infty, r[)} \Psi(u)}{r}<\frac{\Psi(w)}{\Phi(w)} .
$$


Finally for every

$$
\lambda \in \Lambda \subseteq] \frac{\Phi(w)}{\Psi(w)}, \frac{r}{\sup _{u \in \Phi^{-1}(]-\infty, r[)} \Psi(u)}[,
$$

since the weak solutions of the problem (1.1) are exactly the solutions of the equation $I_{\lambda}^{\prime}(u)=0$, then Theorem 2 (with $\tilde{u}=w$ ) and lemma 2 will be guaranteed the conclusion.

Remark 3. According to $F(x, t)=\int_{0}^{t} f(x, \xi) d \xi$ for all $(x, t) \in[a, b] \times \mathbb{R}$, we can consider $F(t)=\int_{0}^{t} f(\xi) d \xi \quad$ for all $t \in \mathbb{R}$. When $f$ is a continuous and nonnegative function then $F \in C^{1}(\mathbb{R})$ and since $F^{\prime}(t)=f(t) \geq 0$ for all $t \in \mathbb{R}$ then $F(t)$ is non-decreasing and so

$$
F^{\theta}=\int_{a}^{b} \sup _{|\xi| \leq \theta} F(\xi) d x=(b-a) \sup _{|\xi| \leq \theta} F(\xi)=(b-a) F(\theta) \text { for all } \theta>0 .
$$

Hence when $\mathrm{f}$ does not depend on $\mathrm{x}$, hypothesis $(i)$ in Theorem 4 becomes the following form:

$$
(I) \frac{F(\theta)}{\theta^{2}}<\frac{2 \Theta F(\delta) \int_{\gamma}^{\frac{b+\gamma}{2}} \alpha(x) d x}{(b-a)\|\alpha\|_{\infty} m_{1} Z \delta^{2}},
$$

and the interval becomes

$$
] \frac{m_{1} Z \delta^{2}}{2 F(\delta) \int_{\gamma}^{\frac{b+\gamma}{2}} \alpha(x) d x}, \frac{\Theta \theta^{2}}{(b-a)\|\alpha\|_{\infty} F(\theta)}[.
$$

Now, we present the following example to illustrate Theorem 4 and Remark 3.

Example 1. Suppose that $f: \mathbb{R} \rightarrow \mathbb{R}$ is continuous and non-negative function and

$$
\alpha(x):= \begin{cases}4 & \text { if } x \in\left[\frac{1}{2}, \frac{3}{4}\right], \\ 0 & \text { if } x \in[0,1]-\left[\frac{1}{2}, \frac{3}{4}\right] .\end{cases}
$$

Also put $K(t)=1+\tanh \sqrt{t}$ for all $t \geq 0$ and therefore we can consider $m_{0}=1$ and $m_{1}=2$. Let $a=0, b=1, \beta=2, \gamma=\frac{1}{2}$ and hence we have $Z=6$ and $\Theta=\frac{2}{9}$. Now, if we assume that $\delta=0.1, \theta=10$ and

$$
\frac{1}{100} \int_{0}^{10} f(\xi) d \xi<\frac{25}{27} \int_{0}^{0.1} f(\xi) d \xi,
$$


then according to Theorem 4 and Remark 3 for each

$$
\lambda \in] \frac{3}{50 \int_{0}^{0.1} f(\xi) d \xi}, \frac{50}{9 \int_{0}^{10} f(\xi) d \xi}[,
$$

problem

$$
\left\{\begin{array}{l}
-\left(1+\tanh \left(\sqrt{\int_{0}^{1}\left|u^{\prime}(x)\right|^{2} d x}\right)\right) u^{\prime \prime}(x)=\lambda \alpha(x) f(u(x)), x \in(0,1), \\
u(0)=0, u(1)=2 u\left(\frac{1}{2}\right)
\end{array}\right.
$$

has at least one non-trivial and non-negative weak solution $u_{0}$ such that $\left|u_{0}(x)\right|<$ 10 for every $x \in[0,1]$.

Remark 4. For example in problem (3.11) we can consider,

$$
f(t):= \begin{cases}100 t^{2} & \text { if } t \leq 0.1 \\ -100 t+11 & \text { if } 0.1<t \leq 0.11 \\ 0 & \text { if } t>0.11\end{cases}
$$

Now we will see that under the appropriate conditions on the function $f$, existence a non-trivial weak solution will be guaranteed for problem

$$
\left\{\begin{array}{l}
-K\left(\int_{a}^{b}\left|u^{\prime}(x)\right|^{2} d x\right) u^{\prime \prime}(x)=\lambda \alpha(x) f(u(x)), \quad x \in(a, b), \\
u(a)=0, u(b)=\beta u(\gamma)
\end{array}\right.
$$

for any $\lambda \in \mathbb{R}^{+}$. In this case the following proposition is a consequence of Theorem 4.

Corollary 1. Assume that $f: \mathbb{R} \rightarrow \mathbb{R}$ is continuous and non-negative function such that

$$
\lim _{\xi \rightarrow 0^{+}} \frac{f(\xi)}{\xi}=+\infty \text { and } \lim _{\xi \rightarrow+\infty} \frac{f(\xi)}{\xi}=0
$$

Then, for each $\lambda \in \mathbb{R}^{+}$, problem (3.12) admits at least one non-trivial and nonnegative solution.

Proof. Suppose that $\lambda>0$ is fixed. Then from $\lim _{\xi \rightarrow 0^{+}} \frac{f(\xi)}{\xi}=+\infty$ we have

$\lim _{\xi \rightarrow 0^{+}} \frac{F(\xi)}{\xi^{2}}=+\infty$ and so $\frac{1}{\lambda}<\frac{2 \int_{\gamma}^{\frac{b+\gamma}{2}} \alpha(x) d x}{m_{1} Z} \frac{F(\delta)}{\delta^{2}}$, for some $\delta>0$ such that sufficiently small.

On the other hand from $\lim _{\xi \rightarrow+\infty} \frac{f(\xi)}{\xi}=0$ we have 
$\lim _{\xi \rightarrow+\infty} \frac{F(\xi)}{\xi^{2}}=0$ and so $\frac{(b-a)\|a\|_{\infty}}{\Theta} \frac{F(\theta)}{\theta^{2}}<\frac{1}{\lambda}$, for some $\theta>0$ with $\delta<$ $\frac{2 \tau \theta}{(1+|\beta|) \sqrt{Z(b-a)}}$ such that sufficiently large. Now we can apply Theorem 4 and the conclusion follows.

Now, we point out the following existence results, as consequences of Theorem 3.

Theorem 5. Suppose that there are $M>0$ and $\theta$ with $\theta m_{0}>2 m_{1}$ such that

$$
0<\theta F(x, \xi) \leq \xi f(x, \xi)
$$

for all $\xi \geq M$ and $x \in[a, b]$. Then for each $\left.\lambda \in \Lambda^{\prime}=\right] 0, \frac{1}{\|\alpha\|_{\infty} F^{s}}[$ where $s=$ $\frac{(1+|\beta|) \sqrt{\frac{2(b-a)}{m_{1}}}}{2}$, problem (1.1) admits at least two distinct non-negative weak solutions.

Proof. Our aim is to apply Theorem 3, to problem (1.1). Put $r=1$ and fixed $\lambda \in \Lambda^{\prime}$. Let $X, \Phi$ and $\Psi$ be as given in the proof of Theorem 4 . We observe that the regularity assumptions of Theorem 3 on $\Phi$ and $\Psi$ are satisfied and also according to proposition 2, the functional $I_{\lambda}$ satisfies the $(P S)$-condition and it is unbounded from below. Since for each $u \in X$ such that $u \in \Phi^{-1}(]-\infty, 1[)$ we have $\|u\|<\sqrt{\frac{2}{m_{1}}}$, hence from (2.1) one has

$$
\begin{gathered}
\frac{\sup _{u \in \Phi^{-1}(]-\infty, r[)} \Psi(u)}{r}=\sup _{\left.\left.u \in \Phi^{-1}(]-\infty, 1\right]\right)} \Psi(u) \\
=\sup _{\left.u \in \Phi^{-1}(]-\infty, 1\right]} \int_{a}^{b} \alpha(x) F(x, u(x)) d x \\
\leq \int_{a}^{b} \alpha(x) \sup _{|\xi| \leq \frac{(1+|\beta|) \sqrt{\frac{2(b-a)}{m_{1}}}}{2}} F(x, \xi) d x \leq\|\alpha\|_{\infty} F^{s}<\frac{1}{\lambda} .
\end{gathered}
$$

From (3.14) we have

$$
\left.\lambda \in \Lambda^{\prime} \subseteq\right] 0, \frac{r}{\sup _{u \in \Phi^{-1}(]-\infty, r[)} \Psi(u)}[.
$$

So all hypotheses of Theorem 3 are verified. Therefore, for each $\lambda \in \Lambda^{\prime}$, the functional $I_{\lambda}$ admits at least two distinct critical points which are, non-negative solutions of problem (1.1) and the proof is complete.

To illustrate Theorem 5, we give the following example. 
Example 2. Let $f:[1,2] \times \mathbb{R} \rightarrow \mathbb{R}$ be the function defined as $f(x, t)=x e^{2 t}$ and $K(t)=(\cos t)^{2}+1$ for all $t \geq 0$. In this case we can consider $m_{0}=1$ and $m_{1}=$ 2. Now we have

$$
F(x, t)=\int_{0}^{t} f(x, \xi) d \xi=\frac{1}{2} x\left(e^{2 t}-1\right) .
$$

Therefore if for example we consider $\theta=5$ then (3.13) is satisfied for $t \geq \frac{5}{2}$ and so according to Theorem 5 for any $\lambda \in] 0, \frac{1}{\|a\|_{\infty} F^{1}}[$ where

$$
F^{1}=\int_{1}^{2} \sup _{|\xi| \leq 1} F(x, \xi) d x=\frac{3\left(e^{2}-1\right)}{4}
$$

the problem

$$
\left\{\begin{array}{l}
-\left(\cos ^{2}\left(\int_{1}^{2}\left|u^{\prime}(x)\right|^{2} d x\right)+1\right) u^{\prime \prime}(x)=\lambda \alpha(x) x e^{2 u(x)}, x \in(1,2), \\
u(1)=0, u(2)=u\left(\frac{3}{2}\right)
\end{array}\right.
$$

has at least two distinct non-negative weak solutions.

\section{ACKNOWLEDGEMENTS}

The author is very thankful for the many helpful suggestions given by the referees who reviewed this paper.

\section{REFERENCES}

[1] G. A. Afrouzi, G. Carisiti, D. Barilla, and S. Moradi, "A variational approach to perturbed threepoint boundary value problems of Kirchhoff-type.” Complex Var. Elliptic Equ., vol. 62, no. 3, pp. 397-412, 2017, doi: 10.1080/17476933.2016.1221948.

[2] G. A. Afrouzi, S. Heidarkhani, and S. Moradi, "Existence of weak solutions for three-point boundary value problems of Kirchhoff-type.” Electron. J. Differ. Equ., vol. 2016, no. 234, pp. 1-13, 2016.

[3] M. Chipot and B. Lovat, "Some remarks on non local elliptic and parabolic problems." Nonlinear Anal., Theory Methods Appl., vol. 30, no. 7, pp. 4619-4627, 1997, doi: 10.1016/S0362546X(97)00169-7.

[4] X. He and W. Ge, “ Triple solutions for second-order three-point boundary value problems." $J$. Math. Anal. Appl., vol. 268, no. 1, pp. 256-265, 2002, doi: 10.1006/jmaa.2001.7824.

[5] X. He and W. Zou, "Infinitely many positive solutions for Kirchhoff-type problems.” Nonlinear Anal., Theory Methods Appl., vol. 70, no. 3, pp. 1407-1414, 2009, doi: 10.1016/j.na.2008.02.021.

[6] S. Heidarkhani, G. A. Afrouzi, and D. O'Regan, " Existence of three solutions for a Kirchhoff-type boundary value problem.” Electron. J. Differ. Equ., vol. 2011, no. 3, pp. 1-11, 2011.

[7] K. Perera and Z. Zhang, "Nontrivial solutions of Kirchhoff-type problems via the Yang index." $J$. Differ. Equations., vol. 221, no. 1, pp. 246-255 , 2006, doi: 10.1016/j.jde.2005.03.006.

[8] S. Shahruz and S. Parasurama, "Suppression of vibration in the axially moving Kirchhoff string by boundary control.” J.Sound Vib., vol. 214, no. 3, pp. 567-575 , 1998, doi: 10.1006/jsvi.1998.1506.

[9] Xiaojie Lin, "Existence of three solutions for a three-point boundary value problem via a threecritical-point theorem." Carpath. J. Math., vol. 31, no. 2, pp. 213-220 , 2015. 
[10] C. O. Alves and G. M. Figueiredo, " Multi-bump solutions for a Kirchhoff-type problem.” Adv. Nonlinear Anal., vol. 5, no. 1, pp. 1-26, 2016, doi: 10.1515/anona-2015-0101.

[11] A. Arosio and S. Panizzi, "On the well-posedness of the Kirchhoff string." Trans. Am. Math. Soc., vol. 348, no. 1, pp. 305-330, 1996, doi: 10.1090/S0002-9947-96-01532-2.

[12] G. Bonanno, " Relations between the mountain pass theorem and local minima." Adv. Nonlinear Anal., vol. 1, no. 3, pp. 205-220, 2012, doi: 10.11515/anona-2012-0003.

[13] G. Bonanno, " A critical point theorem via the Ekeland variational principle." Nonlinear Anal., Theory Methods Appl., vol. 75, no. 5, pp. 2992-3007, 2012, doi: 10.1016/j.na.2011.003.

[14] H. Brezis, Functional Analysis, Sobolev Spaces and Partial Differential Equations. New York: Springer, 2011. doi: 10.1007/978-0-387-70914-7.

[15] C. P. Gupta, " Solvability of a three-point boundary value problems for a second order ordinary differential equation." J. Math. Anal. Appl., vol. 168, no. 2, pp. 540-551, 1992, doi: 10.1016/0022247X(92)90179-H.

[16] G. Kirchhoff, “Vor lesungenüber mathematische Physik: Mechanik.2 ${ }^{\text {te }}$ Aufl .” Leipzig . Teubner (1877) . , 1877.

[17] B. Ricceri, "On an elliptic Kirchhoff-type problem depending on two parameters." J. Glob. Optim., vol. 46, no. 4, pp. 543-549, 2010, doi: 10.1007/s10898-009-9438-7.

Authors' addresses

Mohammad Reza Heidari Tavani

Department of Mathematics, Ramhormoz Branch, Islamic Azad University, Ramhormoz, Iran

E-mail address: m.reza.h56@gmail.com

Mehdi Khodabakhshi

Department of Mathematics, Faculty of Mathematical and Computer Sciences, Shahid Chamran University of Ahwaz, Ahwaz, Iran

E-mail address: m.khodabakhshi11@gmail.com 